\title{
Thermographic measurements in environmental and bio sciences
}

\author{
by C.S. Garbe ${ }^{1}$, U. Schimpf ${ }^{1}$, U. Schurr ${ }^{2}$, B. Jähne ${ }^{1}$ \\ (1) Interdisciplinary Center for Scientific Computing and Institut für Umweltphysik, \\ University of Heidelberg, Im Neuenheimer Feld 368, D-69120 Heidelberg, Germany, \\ E-mail: \{Christoph.Garbe,Uwe.Schimpf,Bernd.Jaehne\}@iwr.uni-heidelberg.de \\ (2) ICG-III (Phytosphäre), Forschungszentrum Jülich GmbH, D-52425 Jülich, Germany
}

\begin{abstract}
In this paper we give an overview of thermographic techniques employed in environmental physics and bio sciences by our research group. Generally, thermography plays an essential role in measuring boundary layer exchange processes. We investigate such processes at the sea surface and on plant leaves. A mid-wave low-noise infrared camera images the temperature distribution directly at the sea surface. By employing digital image sequence analyses important parameters of air-water heat and gas exchange are estimated. The same concept is applied in bio sciences. Here transpiration rates, water content, water flow and heat flux in plant leaves are deducted from thermographic techniques.
\end{abstract}

\section{Introduction}

When studying turbulent transport phenomena, the boundary between different media often plays a predominant role in the underlying transfer process. The reason for this is that due to a suppression of turbulence the boundary represents an effective slow down of the transport. Understanding and quantifying the transport across the boundary layer is thus vital and usually sufficient in characterizing the transport process as a whole.

Conducting measurements in the boundary is often an exceedingly difficult undertaking. In the case of measuring mass transport at the sea surface, the boundary layer extends only a few tenths of micrometers beneath the surface [11] while it is undulated by a few tenths of centimeters by the wave motion. Furthermore it is a well known fact that in order to differentiate between diverse transfer models at the air-water interface temporally and spatially resolved measurements are required in lieu of current point measurements [1]. While spatially resolved measurements of gases still pose strong experimental difficulties, the introduction of heat as a tracer for gas transfer has brought tremendous experimental advances in the field of air sea interactions [8; 10]. Here modern mid-wave low-noise infrared cameras have made temporally and spatially highly resolved measurements feasible for the first time [5]. These measurements can then lead to improving our understanding of atmosphere ocean gas exchange [9].

The application of thermography to botany allows an unobtrusive measurement of thermal properties of plant leaves. Plant leaves have developed a short term thermoregulation also known as transpiration to maintain a temperature most favorable to their metabolism. Here water vapor loss is actively controlled through altering the aperture of pores formed by a complex of cells in the outermost cell layer of the leaf [14]. Apart from their thermoregulation function the pores or stomata represent the main link in between external atmosphere and leaf internal gas space. Due to this integration of many vital transport phenomena in plant leaves, the stomata are highly controlled by internal and external factors. The transpiration of plants and its affecting boundary layer at the leaf have been studied extensively. However, currently available techniques have the major drawback of being point measurements only with long integration times [17].

In this contribution the use of digital image processing techniques and an appropriate statistical analysis will be introduced to estimate the temperature of the water bulk from 
the temperature distribution of the sea surface. By computing the optical flow and material derivative simultaneously, the net heat flux between ocean and atmosphere, a driving force of atmospheric phenomena, is estimated. The same concept is applied in bio sciences. The transpiration rate of plant leaves is measured by the use of passive thermography. Here the temperature of the transpiring leaf is compared to a nontranspiring reference body. Using active thermography the heat capacity of leaves is measured. This heat capacity is equivalent to the water content of the leaf. Digital image processing techniques allow for the analyses of the water velocity in plant leafs, as well as the estimation of net heat flux density at the leaf surface.

\section{Total derivative of sea surface temperature with respect to time}

Thermal images of the sea surface exhibit characteristic fingerprints of the underlying turbulent structures (see Figure 1). This comes about due to a cooling of the interface relative to the bulk due to a net heat flux directed away from the sea surface. Due to turbulences warm water from the bulk is transported into the "cool skin of the ocean". Exposed to a heat flux, the warm water in the interface will then cool down as it travels along the sea surface. This rate of cooling down, equivalent to the total derivative, is important for deriving a number of physical parameters. It can be estimated from digital image processing by simultaneously computing the motion $\vec{f}=(u, v)^{T}$ and the linear parameter of brightness change $c$ [7]. Writing the equation of motion leads to

$$
\frac{d T}{d t}=\frac{\delta T}{\delta t}+\vec{f} \vec{\nabla} T=c \quad \text { or } \quad \vec{d}^{T} \vec{p}=\left(-1, T_{x}, T_{y}, T_{t}\right) \cdot(c, u, v, 1)^{T}=0
$$

in vector notation. Here subscripts indicate the partial derivatives, $T$ is the surface temperature as imaged by the camera, $\vec{d}$ is the data vector and $\vec{p}$ the parameter vector. Equation [1] can be transformed into an overdetermined set of equations by assuming constant parameters in a local spatio-temporal neighborhood and extending the motion constrain over this region. The two dimensional motion vector $\vec{f}$ and the total derivative $c$ can thus be simultaneous estimated in a total least squares sense [7], as is shown in Fig 1.

\section{Sea surface temperature depression}

The temperature depression across the cool skin of the ocean $\Delta T$ is an important parameter for air-sea gas exchange and satellite remote sensing. It is of the order of only $0.1 \mathrm{~K}$ across the aqueous boundary, which extends roughly $1 \mathrm{~mm}$ below the sea surface. In the previous section it was explained that structures in thermal images of the sea surface are produced by warm bulk water impinging on the cooler surface water by turbulent processes. There exists strong experimental evidence for the probability density function (pdf) of these turbulences to be logarithmic normally distributed $[4 ; 15]$. In this model of surface renewal $\Delta T$ can be computed from [16]

$$
\Delta T=\alpha j \sqrt{\tau} \quad \text { with } \quad \tau=t-t_{0}
$$

where $j$ is the net heat flux, $t_{0}$ is the time of surface renewal and $\alpha$ is an expression of material constants. From this equation and the log normal pdf an analytic function for the temperature distribution at the sea surface can be derived $[5 ; 8]$. It is found to be

$$
p\left(T_{\text {surf }}\right)=\frac{|\Delta T|}{(\alpha j)^{2}} \exp \left[\frac{\sigma^{2}}{4}-m\right] \operatorname{erfc}\left[\frac{\sigma}{2}-\frac{m}{\sigma}+\frac{1}{\sigma} \ln \left(\frac{\Delta T}{\alpha J}\right)^{2}\right]
$$


where the parameters $\sigma$ and $m$ describe the log normal distribution of the surface renewal model and erfc is the complimentary error function. From a non linear fit of this analytical function to the surface temperature distribution the parameters $\Delta T$ and $\sigma$ can be computed independently. An example of the fit to field data is shown in Figure 2. This statistical analysis allows to accurately deriving the sought temperature depression $\Delta T$.

\section{Probability density function of surface renewal model}

For gaining insights into the turbulent transport mechanisms of air water heat and gas transfer it is essential to measure the probability density function (pdf) of times in between consecutive surface renewal events. From Equation (2) and the total derivative of it the expression

$$
\tau=\frac{1}{2} \frac{\Delta T}{d / d t \Delta T}
$$

can be derived. In this equation $\Delta T$ is measured from the statistical analysis presented in the previous section and the total derivative from digital image processing as presented in Section 2. Thus $\tau$ can be computed for every pixel of the thermographic sequence. A histogram plot of this data is shown in Figure 2 giving experimental evidence that the pdf of surface renewal can indeed be described very well by a logarithmic normal distribution.

\section{Net heat flux at sea surface}

Similar in principle to estimating $\tau$ in Equation (4) the net heat flux can be computed from Equation (2) and the total derivative of this equation, leading to

$$
j=\frac{\sqrt{2}}{\alpha} \sqrt{\Delta T \frac{d}{d t} \Delta T}
$$

Again, $\Delta T$ and its total derivative are estimated from thermographic sequences as outlined previously. From this equation net heat fluxes can be computed with the frame rate of the IR camera to a spatial resolution also limited by the imager. To validate this technique, measurements were conducted in the Heidelberg Aeolotron, a dedicated windwave flume, and also in situ in the equatorial pacific. The results of computing net heat flux from Equation (5) were compared to those measured from micrometeorological techniques exhibiting excellent agreement (see Figure 3). Modulations of the net heat flux with wave motion could be measured for the first time.

\section{Transpiration rates}

The transpiration of a leaf leads to a latent heat flux, resulting in a cooling of the leaf with respect to its surroundings. It is this change in temperature between a non transpiring reference body and the transpiring leaf than can be used to estimated the transpiration rate $j^{\text {mol }}$ (see Figure 4). For small differential temperatures between the reference body and the leaf $\Delta T$ there exist a linear dependency given by [6]

$$
j^{\text {mol }}=b \cdot k^{\text {leaf }} \cdot \Delta T+X
$$

where $b$ is known from material properties and $k^{\text {leaf }}$ is the heat transfer velocity. The offset $X$ is dependent on the reference body and can be neglected if both reference body and leaf exhibit similar thermal properties. From thermography the transpiration rate of the leaf can be measured spatially resolved, allowing insights into the boundary layer affecting the heat transfer of the leaf. An example of this is shown in Figure 4. 


\section{Water content}

Due to its high water content it can be assumed that the heat capacity of a leaf is only given by this water. Measuring the heat capacity per area $(C / A)$ is thus equivalent to measuring the water content of a leaf. This heat capacity can be estimated from the heat transfer velocity $k^{\text {leaf }}$ as measured in the previous section and a characteristic time constant $\tau$. By using concepts equivalent to those used in Lock-In Thermography [2; 3], the time constant can be measured spatially resolved [12]. By heating the leaf periodically and measuring the phase shift, $\tau$ can be estimated as shown in Figure 5 . Alternatively an incident heat flux can be switched on and the time measured until equilibrium is reached, see Figure 5. The water layer thickness computed from this heat capacity in Figure 5 is in good agreement with microscopic measurements.

\section{Net heat flux and water flow}

By heating up a parcel of water in a leaf and observing its evolution over time, the water flow velocity can be estimated together with the net heat flux (see Figure 6). This can be achieved by a technique similar in principle to that introduced in Section 5 . At a leaf the total derivative can be written as $d / d t T=T_{t}+u T_{x}+v T_{Y}=D \Delta T$ where $\Delta$ indicated the Laplace operator and $D$ the constant of diffusion. By the same computation as presented in Section 2 this total derivative of the temperature is estimated. The net heat flux of the plant leaf is then given as

$$
j=\left(\frac{C}{A}\right) \frac{d}{d t} T=\left(\frac{C}{A}\right) D \Delta T
$$

The heat capacity per area of a plant leaf can be measured by the technique introduced in Section 7. Therefore from this analysis both the net heat flux and the water flow velocity are estimated as presented in Figure 6.

\section{Conclusion}

In this contribution thermographic techniques were presented that allowed estimating parameters of air water heat transfer and the water relations in plant leaves to a high temporal and spatial resolution. Through these techniques insights into the underlying processes and boundary layer effects could be gained. Only through recent advances in low noise mid range infrared cameras combined with modern image processing techniques were these measurements feasible.

\section{REFERENCES}

[1] Broecker, W. S. and Peng, T. H., 1982, Tracers in the Sea: Lamont-Doherty Geological Observatory, Columbia University, Palisades, New York.

[2] Busse, G.. Kunststoffe zerstörungsfrei prüfen. Kunststoffe 90, pp. 212-222. 2000.

[3] Busse, G., Wu, D., and Karpen, W.. Thermal wave imaging with phase sensitive modulated thermography. Journal of Applied Physics 71, pp. 3962-3965. 1992.

[4] Garbe, C. S., Haußecker, H., and Jähne, B., 2001, Measuring the Sea Surface Heat Flux and Probability Distribution of Surface Renewal Events in Gas Transfer at Water Surfaces: Geophysical Monograph, vol. 127, pp. 109-114, American Geophysical Union.

[5] Garbe, C. S., Schimpf, U., and Jähne, B.. Measuring important parameters for airsea heat exchange. Maldague, X. P. and Rozlosnik, A. E.ThermoSense.SPIE 4710, pp. 171-182. 2002. 
[6] Garbe, C. S., Schurr, U., and Jähne, B.. Thermographic measurements of plant leaves. Maldague, X. P. and Rozlosnik, A. E. ThermoSense. 4710, pp. 407-416. 2002. SPIE.

[7] Garbe, C. S., Spies, H., and Jähne, B.. Estimation of surface flow and net heat flux from infrared image sequences. Journal of Mathematical Imaging and Vision . 2002. Kluwer.

[8] Haußecker, H., Schimpf, U., Garbe, C. S., and Jähne, B., 2001, Physics from IR image sequences: Quantitative analysis of transport models and parameters of airsea gas transfer in Gas Transfer at Water Surfaces, Geophysical Monograph, vol. 127, pp. 103-108, AGU.

[9] Jähne, B. and Haußecker, H.. Air-Water Gas Exchange. Annual Reviews Fluid Mechanics 30, pp. 443-468. 1998.

[10] Jähne, B., Libner, P., Fischer, R., Billen, T., and Plate, E. J.. Investigating the transfer process across the free aqueous boundary layer by the controlled flux method. Tellus 41B[2], pp. 177-195. 1989.

[11] Kraus, E.B. and Businger, J.A.,1994, Atmosphere-ocean interaction: Oxford Univ Press.

[12] Kümmerlen, B., Dauwe, S., Schmundt, D., and Schurr, U., 1999, Thermography to Measure Water Relations of Plant Leaves in Handbook of computer vision and applications: Jähne, B., Haußecker, H., and Geißler, P., Eds., 3, chap. 36, pp. 763782, Academic Press.

[13] Lösch, R., 2001, Wasserhaushalt der Pflanzen: Quelle \& Meyer Verlag.

[14] Nobel, P., 1991, Physiochemical and environmental plant physiology: Academic Press.

[15] Rao, K. N., Narasimah, R., and Narayanan, M. B.. The 'bursting' phenomenon in a turbulent boundary layer. Journal of Fluid Mechanics 48, pp. 339-352. 1971.

[16] Soloviev, A. and Schlüssel, P. ,Parameterization of the Cool Skin of the Ocean and the Air-Ocean Gas Transfer on the Basis of Modeling Surface Renewal.JPO 24[6], $1339-46, ' 94$

[17] von Willert, D., Matyssek, R., and Heppich, W., 1995, Experimentelle Pflanzenökologie: Georg Thieme Verlag, Stuttgart.
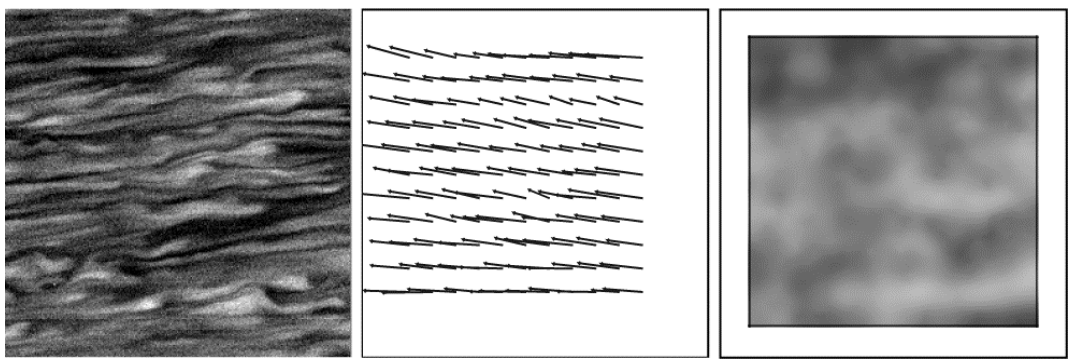

Fig. 1. Image of a thermographic sequence (left), the $2 D$ flow field (middle) and the total derivative of the temperature with respect to time (right) 

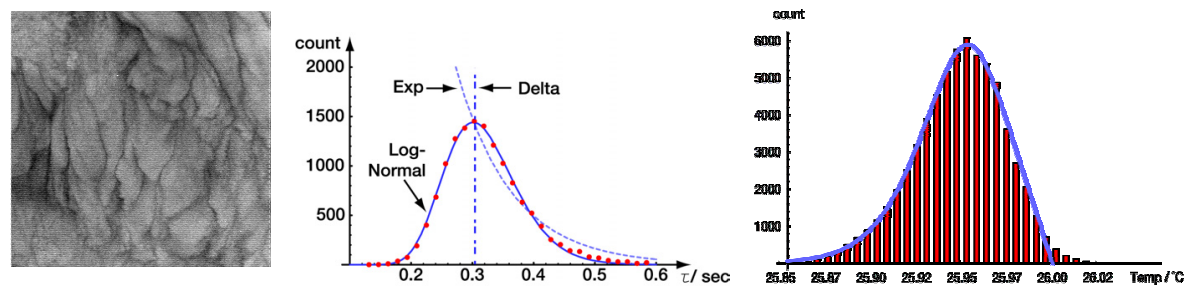

Fig. 2. An image of the sea surface temperature distribution and the resulting Histogram. The analytical function fitted to the data shows excellent agreement. The log normal pdf also shows much better agreement with the data than exponential or delta distribution

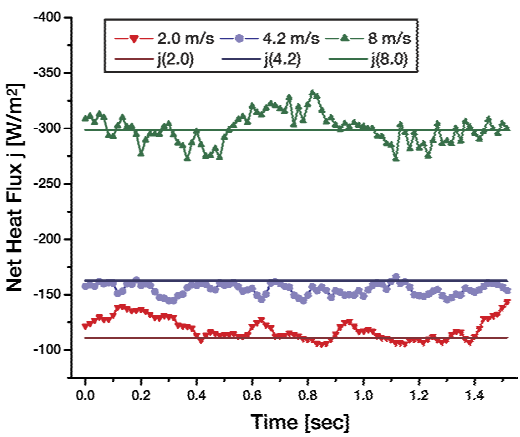

a)

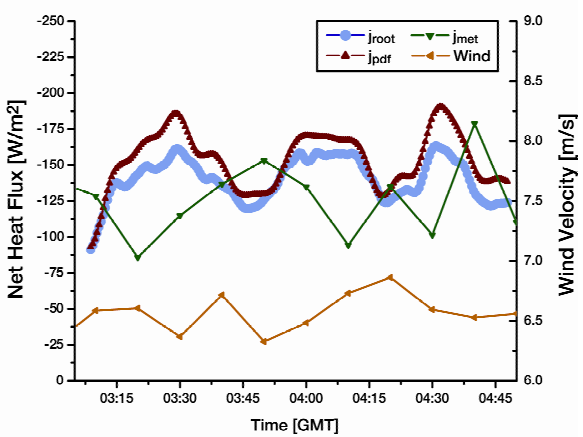

b)

Fig. 3. Comparison of the estimated net heat flux with the ground truth data in a). Comparison of the net heat flux with micro meteorological measurements

Stomata

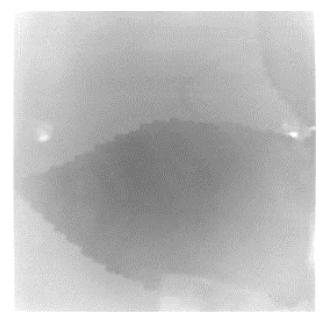

Stomata

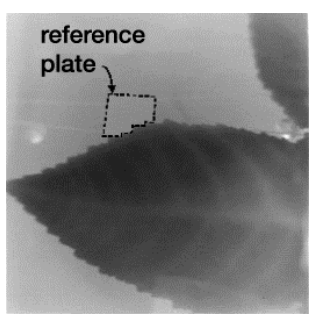

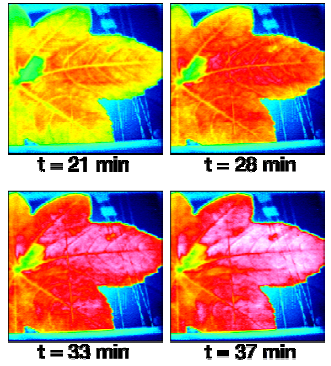

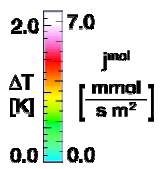

Fig. 4. Images of a plant leaf with closed and opened stromata as well as spatially and temporally resolved estimates of transpiration rates 

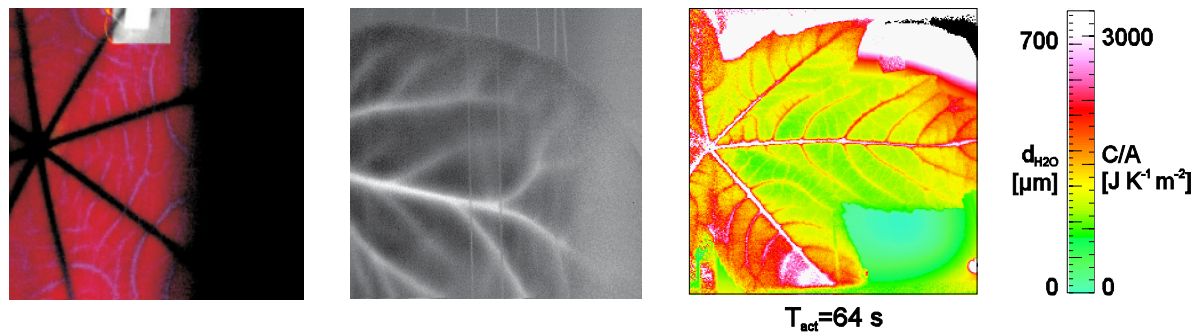

Fig. 5. From left to right: Time lag in response to heating, Phase shift of periodic heating and the derived water layer thickness
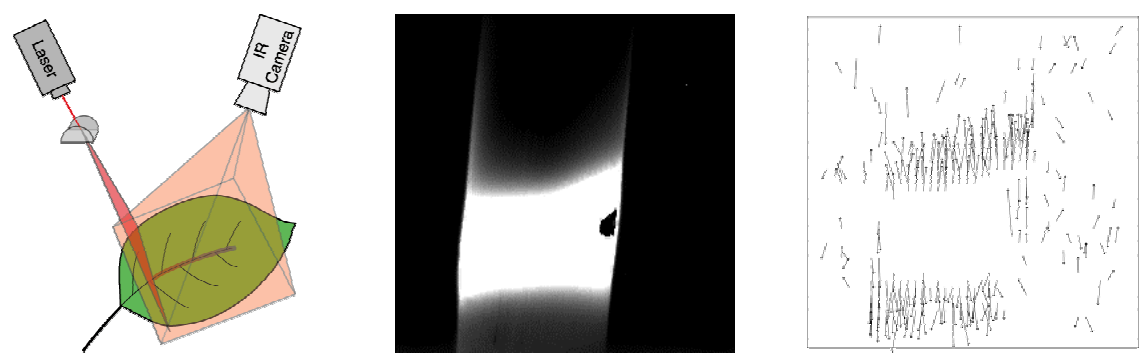

Fig. 6. From left to right: The experimental set-up for heating up a water parcel in the leaf, an image from the thermographic sequence and an image of the estimated water flow 